\title{
Turbulence Imaging Simulation Based on Electrically Tunable Polymer Dispersed Liquid Crystals Cell
}

\author{
Hui LI $^{1,2,4}$, Fan PAN ${ }^{3}$, Yuntao WU ${ }^{1,2}$, Yanduo ZHANG ${ }^{1,2}$, Xiaolin XIE $^{4}$ \\ ${ }^{1}$ School of Computer Science and Engineering, Wuhan Institute of Technology, Wuhan 40073, P.R. China \\ ${ }^{2}$ Hubei Key Laboratory of Intelligent Robot, Wuhan 40073, P.R. China \\ ${ }^{3}$ College of Post and Telecommunication, Wuhan Institute of Technology, Wuhan 40073, P. R. China \\ ${ }^{4}$ School of Chemistry and Chemical Engineering, Huazhong University of Science and Technology, Wuhan 40073, \\ P.R. China
}

crossref http://dx.doi.org/10.5755/j01.ms.25.2.19136

Received 27 September 2017; accepted 30 March 2018

\begin{abstract}
In this study, the scattering properties of polymer dispersed liquid crystals (PDLC) and its optical features for use in turbulence image simulation are presented. A PDLC cell composed of a liquid crystal/monomer mixture with two indium tin oxide glass substrates, $55 \mathrm{wt} . \%$ E7 and $45 \mathrm{wt} . \%$ NOA65, is proposed. And this mixture had a thickness of $10 \mu \mathrm{m}$. With respect to its scattering properties, it was hypothesized that the proposed PDLC cell could be utilized as an electrically tunable turbulence imaging simulator. The measured results, two dimensional images of the transmission light, under voltages from 0 Vrms to 10 Vrms showed that the PDLC film could effectively simulate turbulence situation of a point light source under atmosphere turbulence condition to a certain extent. This PDLC film had a great potential for application in turbulence imaging simulation.

Keywords: polymer dispersed liquid crystals, scattering properties, turbulence image simulator.
\end{abstract}

\section{INTRODUCTION}

Polymer dispersed liquid crystal (PDLC) is composed of a polymer and liquid crystals [1]. In recent years, PDLC has been applied widely in many fields, such as displays, holography, optical shutters, imaging correctors, and intelligent buildings [2-8]. Usually, there are several basic methods of preparation, temperature induced phase separation (TIPS), solvent induced phase separation (SIPS), polymerization induced phase separation (PIPS), and microencapsulation process (MP), respectively [9]. Amongst them, the most common and effective preparation method is PIPS. Many characteristics of PDLCs have been studied. In particular, its photoelectric characteristics are still a focus of attention and the scattering properties of the PDLC film are very important features. Scattering is caused by the spatial variation of the refractive index in the liquid crystal/polymer mixture. The main cause of formation is that the refractive index between the liquid crystal and polymer networks is mismatched, as is that between the liquid crystal domains $[10,11]$. In consequence, there have been many investigations of the formation mechanism and of the consequent scattering characteristics $[10,11]$. Furthermore, there are also many applications of PDLC films in different fields that use its scattering characteristics $[6,7]$. However, related applications in the field of imaging systems are worthy of further research, especially for turbulence imaging simulation. As it is well-known, the liquid crystal is widely utilized in the simulation of atmospheric turbulence $[12,13]$. But, there is still no enough study on the simulation with use of PDLC. The motivation for this

\footnotetext{
* Corresponding author. Tel.: +86-27-87992007; fax: +86-27-87992007 E-mail address: lihui00317@163.com (H. Li)
}

work was to apply a PDLC cell to simulate the twodimensional image of a point light source under the condition of atmospheric turbulence.

In general, turbulent images appear under environments of atmospheric turbulence [14, 15]. In order to study the basic principles, many kinds of methods, including software algorithms and hardware implementations, have been proposed. However, the common problems amongst these algorithms and hardware implementations are the large complex computational requirements, which are very costly. Sometimes, multiple iterations are required. They also are inappropriate for use in the real-time simulation and analysis. In order to solve the problem, many fast algorithms and simple hardware implementations have been proposed $[16,17]$. But there is still room to develop efficient simple methods for simulating turbulence images. Because of the birefringence feature of liquid crystals, a number of hardware implementations based on liquid crystal devices have been proposed $[12,13]$. As a result of the scattering properties of PDLCs, it is feasible to produce turbulence images based on PDLC films. In the present investigation, the scattering properties of PDLC films and the influence of its formation mechanism on its capability to simulate turbulence images are discussed and the electricallycontrolled features have been studied in details. Compared to the traditional methods, the proposed approach can give an intuitive result without large computations. It has several advantages, such as low cost, being electrically controlled and being relatively simple.

The second section presents the related theory about PDLC cells and turbulence imaging. The third section describes the fabrication procedure for the proposed PDLC cells and presents some interesting experimental results 
that are used to discuss its scattering properties in turbulence image simulators and present other classic electro-optical features. In conclusion, use of the proposed PDLC cells in turbulence image simulators is considered.

\section{RELATED THEORY}

Chemical potential can be utilized to explain the phase separation phenomenon in PDLCs. The chemical potential of the liquid crystal and the monomers are $\mu_{L C}$, and $\mu_{M}$, respectively [18]:

$$
\left\{\begin{array}{l}
\mu_{L C}=\mu_{L C}^{0}+K T \ln \frac{N_{L C}}{N_{L C}+N_{M}} \\
\mu_{M}=\mu_{M}^{0}+K T \ln \frac{N_{M}}{N_{L C}+N_{M}}
\end{array},\right.
$$

where $L C$ means liquid crystal; $M$ represents monomers; $\mu_{L C}^{0}$ is the chemical potential of pure LC state; $\mu_{M}^{0}$ is the chemical potential of pure monomers state; $N_{L C}$ denotes the number of liquid crystal molecules in the mixture, $N_{M}$ means the number of monomers molecules in the mixture, $K$ is Boltzmann's constant, and $T$ means absolute temperature.

When thermal equilibrium occurs in the PDLC film, the chemical potential of each component in the film is equal. In areas of strong light intensity, the monomers are polymerized by the external UV light, the monomers gradually become polymer, and the number of monomers in this region obviously decreases. Due to the decrease in the chemical potential of the monomer, according to Eq. 1, the monomers diffuse from the weak light intensity region to the strong light intensity region, based on the principle of thermal equilibrium. In this way, the chemical potential of the monomers compensates. Conversely, in the areas with strong light intensity, because the number of monomers decreases, the chemical potential of the liquid crystal increases in accordance with Eq. 1 and the liquid crystal migrates from the region of strong light intensity to the area of weak light intensity, based on the principle of thermal equilibrium. Correspondingly, the chemical potential of the liquid crystal compensates. As a result, phase separation arises and the PDLC film is prepared.

The working principle of PDLCs for light modulation is shown in Fig. 1. In the voltage-off state, as shown in Fig. 1 a, the liquid crystal droplets are randomly oriented. The effective refractive index $n_{\text {eff }}$ seen by the light will be different to that of the polymer networks $n_{p}$. Hence, the incident light is scattered mainly in the forward direction or is reflected. In the voltage-on state, the LC molecules are reoriented along the electric field direction, as shown in Fig. 1 b. Therefore, the ordinary refractive index of LC $n_{o}$ is the refractive index seen by the light, and usually $n_{p}$ is very close to $n_{o}$. So light passes through the PDLC layer with high transmission and less scattering.

The PDLC film has a very special feature of an optical shutter. Without the externally-applied voltage, the liquid crystals in the PDLC film tend to align parallel to one direction in a single droplet. However, every liquid crystal droplet has its own direction. Thus, there are very many different refractive indexes at those interfaces and they all do not match with each other.

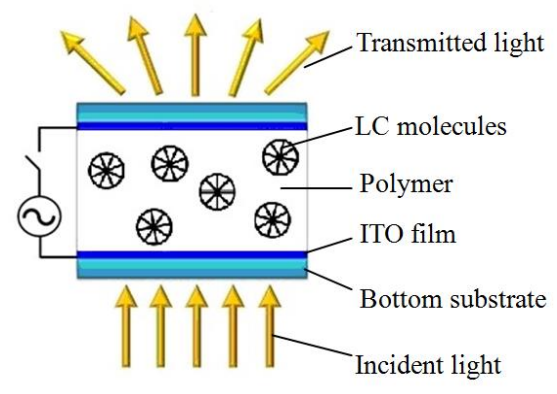

a

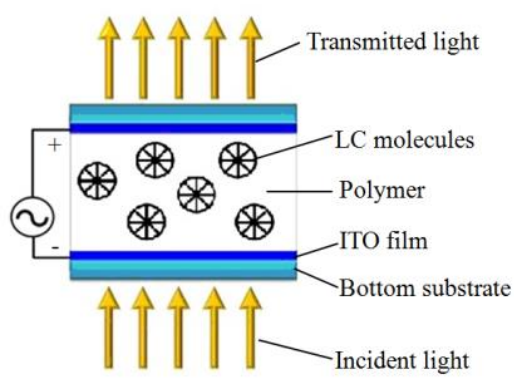

b

Fig. 1. Operation mechanism of PDLC for light modulation, where the director of liquid crystal is radial type: a-in the state of power off; $b$-in the state of power on, and the direction of applied voltage is as shown

Those interfaces effectively scatter the incident light. Under this situation, the PDLC film is an opaque state. With an external applied voltage, the liquid crystal molecules first rotate, then orientate perpendicular to surfaces of both top and bottom substrates. The vertical incident light sees the same refractive index of liquid crystals and, as a result, those interfaces all disappear and light passes through the PDLC film. Then the PDLC film becomes transparent. The transmittance of the PDLC cell can be controlled by the applied external voltage. The formula of the transmittance in PDLC film is [18]:

$$
T\left(E_{\text {ext }}\right)=T_{A} \exp \left[-\alpha\left(E_{\text {ext }}\right)\right] \text {, }
$$

where $\alpha\left(E_{e x t}\right)=\chi \sigma\left(E_{e x t}\right) d$, and $T_{A}$ is the maximum transmittance, $\chi$ means the density number of the liquid crystal droplets, $\sigma$ denotes the average scatter cross section, $d$ is the thickness of the PDLC film, and $E_{\text {ext }}$ is the applied external voltage.

It is well known that atmospheric disturbance is the main cause of turbulence image formation, which leads to very poor image resolution [12]. The reason is that the refractive index of the atmosphere can change randomly in both space and time. The results in similarly random changes in the optical path differences in the atmosphere medium, when a plane wave from a very far distant source is incident on the film. As the view of a reference star passing through an atmosphere is distorted during the propagation in atmosphere, because the plane wavefront is distorted and changes in this non-planar wavefront can be regarded as optical aberration. 
Similarly, a large burst of heated air will be continuously and randomly dissipated into a small volume of air at uniform temperature, in the form of turbulence eddies. The refractive indices in the turbulent eddies are all the same. Based on the Kolmogorov theorem, the formula of spatial power spectral density is [20]:

$\Phi_{\varphi}(k)=\frac{0.023 k^{-11 / 3}}{r_{0}^{5 / 3}}$,

where $r_{0}$ is a normalization factor with units of length that gives the correct dimensional of the power spectrum; $k$ is the spatial wave-number vector. When the spatial wavenumber vector satisfies $\frac{2 \pi}{L_{0}} \leq k \leq \frac{2 \pi}{l_{0}} \quad\left(L_{0}\right.$ means the maximum distance, $l_{0}$ means the minimum distance), compliance with the Kolmogorov theorem is confirmed [20]. Otherwise, the Kolmogorov theorem is not confirmed [20].

\section{EXPERIMENTS AND DISCUSSION}

In this study, the PDLC film was produced by using UV light. During the procedure, liquid crystal, E7 from the Merck Co., and polymer, NOA65 from Norland Co., were utilized. The chosen reason was that refractive index $n_{\mathrm{p}}$ of the polymer was very close to refractive index $n_{\mathrm{o}}$ of the liquid crystal. For this sample, the mass ratio of those two materials, 55:45, was chosen. The main fabrication flow of the PDLC cell was as follows: first, the liquid crystal and the monomers were mixed according to the mass ratio of 55 wt.\%: 45 wt. $\%$ in a glass bottle with the use of an electric balance. An ultrasonic shaker was utilized to agitate it to ensure uniform mixing. Next, an empty cell, composed of two Indium Tin Oxides (ITO) substrates and spacers was prepared. The thickness of the PDLC film was $10 \mu \mathrm{m}$. The mixture of liquid crystal-polymer was injected into the empty cell by capillary effect. Finally, AB glue was utilized to seal the liquid crystal cell. Under UV irradiation, the sealed liquid crystal cell with a PDLC film was obtained.



Fig. 2. Experimental setup

A testing arrangement was set up, as shown in Fig. 2. In order to acquire a point light source, an optical stop was used. It was set in front of the light source. The PDLC cell was between the light source and CCD (Charge Coupled Device). Light was passed through the PDLC cell and fell on the CCD. The CCD was 3 Megapixel in resolution, $1 / 2$ inch in thickness, and $6.4 \mathrm{~mm} \times 4.8 \mathrm{~mm}$ in area, and was connected to a PC. A tunable external applied voltage (0 Vrms - $10 \mathrm{Vrms})$ at $1 \mathrm{KHz}$ was used to drive the PDLC cell.

An optical image of the proposed PDLC cell was acquired by a Polarizing Optical Microscope (POM), as shown in Fig. 3. The test condition for this acquired image was under $2 \mathrm{Vrms}$. The scattering properties of the PDLC can be explained by Mie scattering theory. Liquid crystal droplets in the PDLC can have several molecular alignment modes. In Fig. 3, liquid crystal is in radial mode. The scattering properties of PDLC can be affected by several important parameters, such as the size of the liquid crystal droplets, the mass ratio between the liquid crystals and the monomer, the degree of matching between the refractive index of the liquid crystal $n_{\mathrm{o}}$ and the refractive index of the polymer $n_{\mathrm{p}}$, the thickness of the PDLC film, and so on [7]. For the proposed PDLC cell, the distance between two liquid crystal droplets is a very important factor that affects scattering properties. If the distance is too small, the liquid crystal droplets easily connect with each other. On the other hand, if the distance is too large, the density of liquid crystals will be low. If the density of liquid crystal increases, the number of light scattering points in the PDLC film increases and scattering phenomenon will become more evident. Conversely, if the density of liquid crystal decreases, the number of light scattering points in the PDLC film decreases and the scattering phenomenon is weaker. As shown in Fig. 3, the distance between liquid crystal droplets is relatively balanced and there are only narrow spaces between the liquid crystal droplets in the PDLC film. The other important factor affecting the scattering properties of the proposed PDLC film is the diameter of the liquid crystal droplets. If the weight ratio of liquid crystal in the PDLC film is small, the liquid crystal droplets formed in it have only a small diameter. Correspondingly, if the weight ratio of liquid crystal is large, the time for the liquid crystal to separate out of PDLC is sufficiently long that the liquid crystal droplets then formed have a large diameter. In Fig. 3, diameter of liquid crystals visible in the mixture is generally small and is several micrometers by comparison with the scale bar of $50 \mu \mathrm{m}$ also shown. This value is appropriate to obtain better scattering properties from the PDLC.

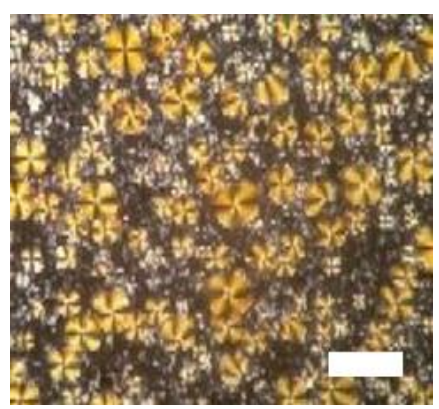

Fig. 3. POM image of PDLC film, and the scale bar is about $50 \mu \mathrm{m}$

Based on the principle of Gaussian beam, the spot size of Gaussian beam is $w(z)=w_{0} \sqrt{1+\left(\frac{z}{z_{0}}\right)^{2}}$, where $z_{0}=\frac{\pi w_{0}^{2}}{\lambda}$ is beam waist radius. In the far field, $w(z)$ can be approximated as, $w(z)=\frac{w_{0}}{z_{0}} z=\frac{\lambda}{\pi w_{0}} z$ [21]. From the 
approximate formula, $w(z)$ and $z$ has a linear relationship. Thus, the divergence angle of PDLC film in the far field is $\theta \approx \tan \theta=\frac{w}{z}=\frac{\lambda}{\pi w_{0}}[21]$.

In order to analyze the scattering feature of PDLC, a red laser, $633 \mathrm{~nm}$, was utilized in the following experiment. The experiment setup in Fig. 2 was still utilized to measure the divergence angle of PDLC film. The incident light source was replaced as a red laser. A CCD was used to acquire the measurement data. In the experiment, the information of transmission light can be obtained. In Fig. 4 there are two dimensional images of spot profiles under different applied voltages. From the acquired experimental results, it was found that the scattering of light beam varied with the applied voltage. As the voltage increased, the scattering decreased.

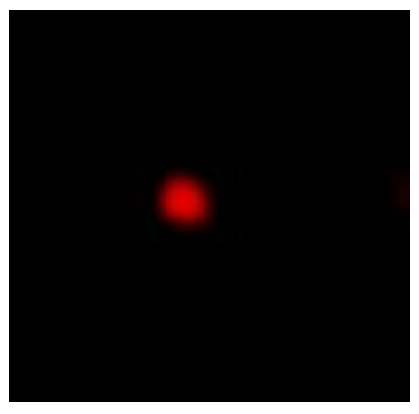

a



$\mathrm{c}$

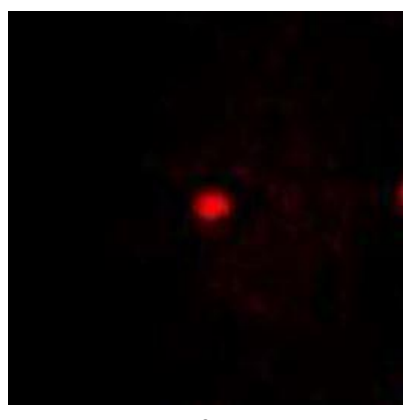

e

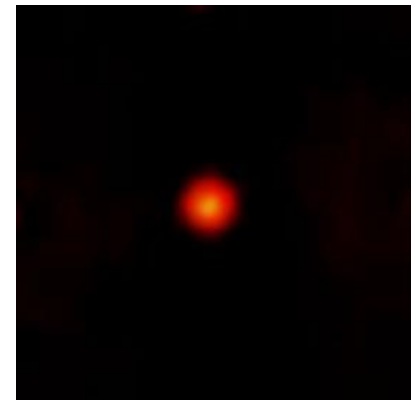

b



d

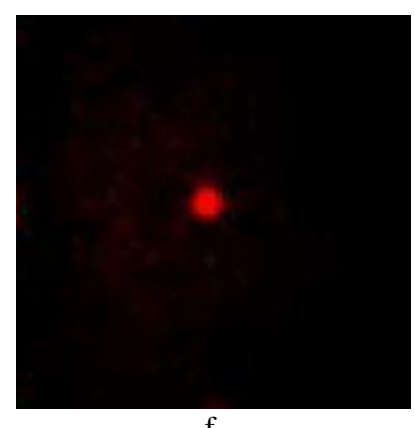

f
Fig. 4. Two dimensional images of the transmission light under different applied voltage: $a-0$ Vrms; $b-2$ Vrms; c-4 Vrms; d-6 Vrms; e-8 Vrms; f-10 Vrms

The divergence angle of PDLC film can be computed by the above mentioned theory. And the formula $\theta \approx \tan \theta=\frac{w}{z}=\frac{\lambda}{\pi w_{0}}$ is used [21]. Table 1 shows the final result. The scattering decreases and the transmission rate increases with the changes of applied voltage.
Next, a verification measurement was set up, as shown in Fig. 2. In order to present clearly the final results of this verfication result, a parallel white light (The direction of parallel is along the main optical axis) was used as a light source and an optical stop was used to generate a point light source. The PDLC cell was set in front of the CCD, which was used as pupil. Turbulence is the main cause of refractive indices in atmospheric media that are mismatched. An electrical resistor was heated for 3 hours to simulate atmospheric thermal disturbance and, in this way, a distorted wavefront could be obtained. With the respect to the scattering properties of the PDLC cell mentioned above, simulation of a two-dimensional image of the point light source was feasible.

Table 1. The divergent angle of PDLC under different applied voltage

\begin{tabular}{|c|c|}
\hline Applied voltage, Vrms & Divergent angle, degree \\
\hline 0 & 1.9 \\
\hline 2 & 4.5 \\
\hline 4 & 2.1 \\
\hline 6 & 1.5 \\
\hline 8 & 0.8 \\
\hline 10 & 0.2 \\
\hline
\end{tabular}

In Fig. 5, two-dimensional image of a point light source was acquired by $\mathrm{CCD}$, which was connected directly to a PC. The acquired data were analyzed as follows. At 0 Vrms, the PDLC cell was opaque because it was without any driven voltage. Under this situation, when light was passed through the cell, the scattering phenomenon was observed. As the incident light was a point light source, the ideal result on CCD should be a Gaussian wave. However, two-dimensional image produce by CCD did not have a very clear image. Based on the above-mentioned theory in Section 2, at this moment the PDLC cell exhibited a marked scattering property and the light had changed because of scattering. As presented in Fig. 5 a, there was an evident turbulence effect. That behavior is very close to the real situation of atmospheric turbulence. Compared to the traditional methods, the proposed approach gives an intuitive result without large computations [12-14]. When the external applied voltage increases, the degree of scattering becomes weak. At $2 \mathrm{Vrms}$, the transmitted light had weaker scattering properties, as shown in Fig. $5 \mathrm{~b}$ and the transmitted light had a focusing feature. With increasing external applied voltage, the degree of matching between refractive index of liquid crystal and refractive index of polymer was much higher. This trend agrees with the above mentioned scattering feature measurement of the proposed PDLC film. Thus, the transmitted light gradually had a focusing spot, while when the externally applied voltage was up to the saturation value of liquid crystal rotation, the focusing spot was relatively clear, as shown in Fig. $5 \mathrm{f}$. The presented two-dimensional image is analogous to a Gaussian wave. The acquired data in Fig. 5 can confirm the abovementioned theory in Section 2 to a certain extend. However, the result presented here is only preliminary. That is becasue the formation mechanism of the turbulent image given by this PDLC cell is still complicate and many factors can affect simulation result. Nevertheless, 
this work demonstrates a feasible approach to realize this simulation without complex iteration-computation.



a

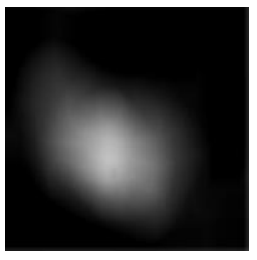

d

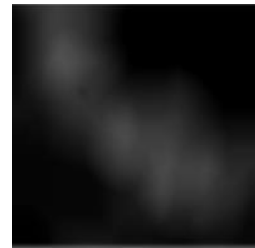

b

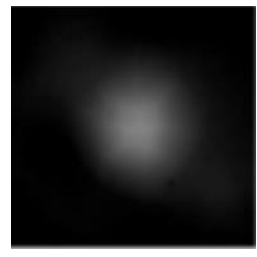

e

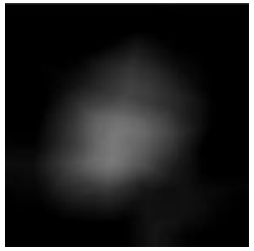

c

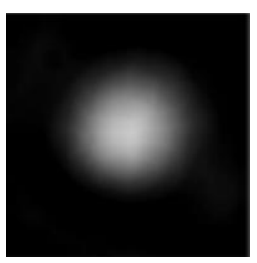

f
Fig. 5. The two dimensional images acquired by CCD under different external applied voltages: a-0 Vrms; $\mathrm{b}-2 \mathrm{Vrms} ; \quad \mathrm{c}-4 \mathrm{Vrms} ; \mathrm{d}-6 \mathrm{Vrms} ; \mathrm{e}-8 \mathrm{Vrms}$; f- 10 Vrms

\section{CONCLUSIONS}

In this study, a PDLC cell was composed of 55 wt.\% E7 and 45 wt.\% NOA65. It is an electrically-controlled device. Its phase separation and scattering capability have been evaluated. A method by using the proposed PDLC cell for simulating atmospheric turbulence is proposed. In measurements, two-dimensional images acquired by the CCD have been presented as confirmation of the proposed hypothesis. The measured results showed that this PDLC cell could be seen as the simulation-results of twodimensional images for a point light source under conditions of atmospheric turbulence. Compared to conventional methods, the proposed approach has several advantages, such as, low cost, less computation, and relatively simplicity. There is still space for further development, considering the wide range of factors, such as, liquid crystal orientation at the liquid crystal-polymer interfaces, that can affect the final result.

\section{Acknowledgments}

This work was supported by a grant from National Natural Science Foundation of China (51703071), the Research Foundation of the Education Bureau of Hubei Province, China (D20171504), and the Open Foundation for the Hubei Key Laboratory of Intelligent Robot (HBIR 201805).

\section{REFERENCES}

1. Ji, H., Kim, J.H., Kumar, S. Electrically Controllable Microlens Array Fabricated by Anisotropic Phase Separation from Liquid-Crystal and Polymer Composite Materials Optics Letters 28 (13) 2003: pp. 1147-1149. https://doi.org/10.1364/OL.28.001147

2. Woltman, S.J., Eakin, J.N., Crawford, G.P., Žumer, S. Holographic Diffraction Gratings Using Polymer-Dispersed Ferroelectric Liquid Crystals Optics Letters 31 (22) 2006: pp. 3273-3275.

https://doi.org/10.1364/OL.31.003273
3. Woltman, S.J., Eakin, J.N., Crawford, G.P., Žumer, S. Electro-Optical Investigations of Holographic-PolymerDispersed Ferroelectric Liquid Crystals Journal of the Optical Society of America A 24 (12) 2007: pp. $3789-3799$.

https://doi.org/10.1364/JOSAA.24.003789

4. Chen, H., Liang, H., Lai, W.H., Li, C.C., Wang, J., Zhou, J., Lin, T.H., Khoo, I.C., Li, J. A 2D/3D Switchable Directional-Backlight Autostereoscopic Display Using Polymer Dispersed Liquid Crystal Films Journal of Display Technology 12 (12) 2016: pp. 1738 - 1744. https://doi.org/10.1109/JDT.2016.2613121

5. Wang, K., Zheng, J., Gao, H., Lu, F., Sun, L., Yin, S., Zhuang, S. Tri-Color Composite Volume H-PDLC Grating and its Application to 3D Color Autostereoscopic Display Optics Express 23 (24) 2015: pp. 31436-31445. https://doi.org/10.1364/OE.23.031436

6. Song, S., Jeong, J., Chung, S., Jeong, S., Choi, B. Electroluminescent Devices with Function of Electro-Optic Shutter Optics Express 20 (19) 2012: pp. 21074-21082. https://doi.org/10.1364/OE.20.021074

7. Hsieh, C.T., Ding, P.R., Liao, C.H., Su, W.C., Huang, C.Y., Lin, C.H., Lo, K.Y., Tien, C.J., Hsu, T.F. Polarization-Independent Distortion Corrector Fabricated Using Polymer-Dispersed Liquid Crystals Applied Optics 53 (3) 2014: pp. 383-387. https://doi.org/10.1364/AO.53.000383

8. Zheng, J., Sun, G., Jiang, Y., Wang, T., Huang, A., Zhang, Y., Tang, P., Zhuang, S., Liu, Y., Yin, S. H-PDLC Based Waveform Controllable Optical Choppers for FDMF Microscopy Optics Express 19 (3) 2011: pp. 2216-2224. https://doi.org/10.1364/OE.19.002216

9. Jamil, M., Ahmad, F., Rhee, J.T., Jeon, Y.J. NanoparticleDoped Polymer-Dispersed Liquid Crystal Display Current Science 101 (12) 2011: pp. $1544-1552$.

10. Geis, M.W., Bos, P.J., Liberman, V., Rothschild, M. Broadband Optical Switch Based on Liquid Crystal Dynamic Scattering Optics Express $24(13)$ 2016: pp. $13812-13823$. https://doi.org/10.1364/OE.24.013812

11. Loŭko, V.A., Konkolovich, A.V., Miskevich, A.A. Using the Wentzel-Kramers-Brillouin Method to Analyze the Transmission and Reflection of Light by a Layer of LiquidCrystal Drops Dispersed in a Polymeric Matrix Journal of Optical Technology 78 (7) 2011: pp. 455-459. https://doi.org/10.1364/JOT.78.000455

12. Cao, Z., Mu, Q., Hu, L., Li, D., Liu, Y., Jin, L., Xuan, L. Correction of Horizontal Turbulence with Nematic Liquid Crystal Wavefront Corrector Optics Express $16(10)$ 2008: pp. 7006-7013. https://doi.org/10.1364/OE.16.007006

13. Hu, L., Xuan, L., Cao, Z., Mu, Q., Li, D., Liu, Y. A Liquid Crystal Atmospheric Turbulence Simulator Optics Express 14 (25) 2006: pp. 11911-11918. https://doi.org/10.1364/OE.14.011911

14. Wu, C., Ko, J., Davis, C.C. Imaging Through Strong Turbulence with a Light Field Approach Optics Express 24 (11) 2016: pp. 11975 - 11986. https://doi.org/10.1364/OE.24.011975

15. Shi, D., Fan, C., Zhang, P., Shen, H., Zhang, J., Qiao, C., Wang, Y. Two-Wavelength Ghost Imaging Through Atmospheric Turbulence Optics Express $21(2)$ 2013: pp. 2050-2064. https://doi.org/10.1364/OE.21.002050 
16. Gilles, L., Ellerbroek, B.L. Real-Time Turbulence Profiling with a Pair of Laser Guide Star Shack-Hartmann Wavefront Sensors for Wide-Field Adaptive Optics Systems on Large to Extremely Large Telescopes Journal of the Optical Society of America A 27 (11) 2010: pp. A76-A83. https://doi.org/10.1364/JOSAA.27.000A76

17. Schöck, M., Le Mignant, D., Chanan, G.A. Wizinowich, P.L., van Dam, M.A. Atmospheric Turbulence Characterization with the Keck Adaptive Optics Systems. I. Open-Loop Data Applied Optics 42 (19) 2003: pp. $3705-3720$. https://doi.org/10.1364/AO.42.003705

18. Ferrari, J.A., Dalchiele, E.A., Frins, E.M., Gentilini, J.A., Perciante, C.D., Scherschener, E. Effect of Size Polydispersity in Polymer-dispersed Liquid-Crystal
Films Journal of Applied Physics 2008: pp. 084505-1-084505-4. https://doi/10.1063/1.2903063

19. Beckers, J.M. Adaptive Optics for Astronomy: Principles, Performance, and Applications Annual Reviews of Astronomy and Astrophysics 31 1993: pp. 13-62. https://doi/abs/10.1146/annurev.aa.31.090193.000305

20. Boreman, G.D., Dainty, C. Zernike Expansions for NonKolmogorov Turbulence Journal of the Optical Society of America A 13 (3) 1996: pp. 517-522. https://doi.org/10.1364/JOSAA.13.000517

21. Pedrotti, F.L., Pedrotti, L.M., Pedrotti, L. S. Introduction to Optics Pearson Addison Wesley, San Francisco, 2007. 